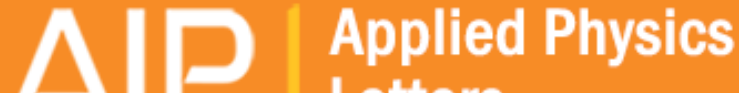 Letters
}

An ionic liquid-gated polymer thin film transistor with exceptionally low "on" resistance

Saud A. Algarni, Talal M. Althagafi, Patrick J. Smith, and Martin Grell

Citation: Applied Physics Letters 104, 182107 (2014); doi: 10.1063/1.4875746

View online: http://dx.doi.org/10.1063/1.4875746

View Table of Contents: http://scitation.aip.org/content/aip/journal/apl/104/18?ver=pdfcov

Published by the AIP Publishing

\section{Articles you may be interested in}

Electrolyte-gated polymer thin film transistors making use of ionic liquids and ionic liquid-solvent mixtures J. Appl. Phys. 117, 112809 (2015); 10.1063/1.4913835

Analyzing the influence of negative gate bias stress on the transconductance of solution-processed, organic thinfilm transistors

J. Appl. Phys. 116, 074507 (2014); 10.1063/1.4893317

Direct patterning of solution-processed organic thin-film transistor by selective control of solution wettability of polymer gate dielectric

Appl. Phys. Lett. 102, 153305 (2013); 10.1063/1.4802499

Enhanced carrier mobility and electrical stability of n-channel polymer thin film transistors by use of low-k dielectric buffer layer

Appl. Phys. Lett. 99, 173303 (2011); 10.1063/1.3655680

Independently driven four-probe method for local electrical characteristics in organic thin-film transistors under controlled channel potential

Rev. Sci. Instrum. 82, 093902 (2011); 10.1063/1.3637489

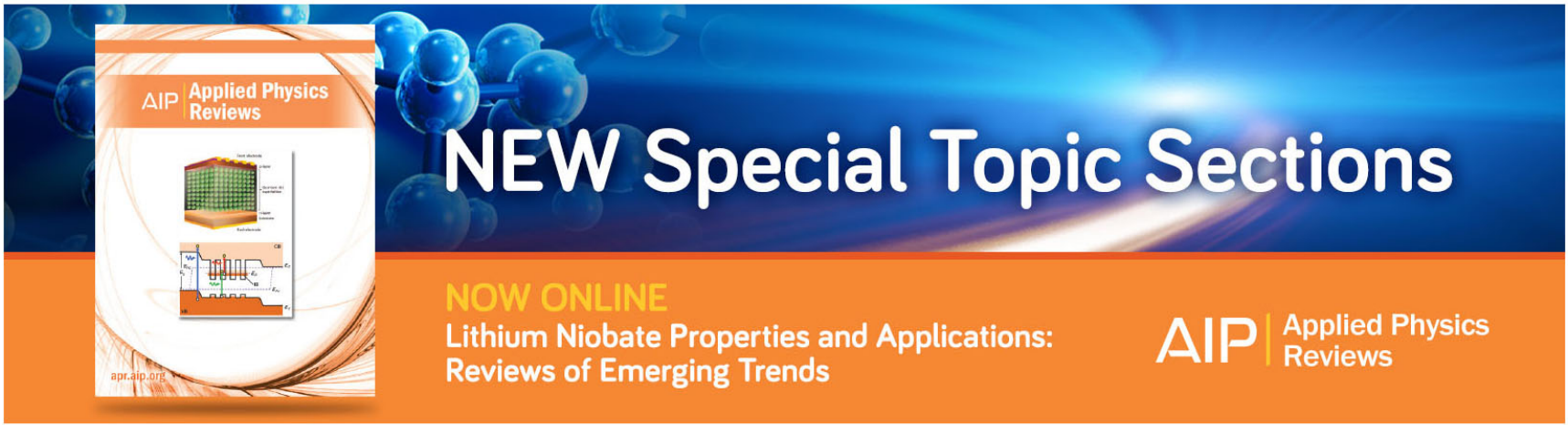




\title{
An ionic liquid-gated polymer thin film transistor with exceptionally low "on" resistance
}

\author{
Saud A. Algarni, ${ }^{1, a)}$ Talal M. Althagafi, ${ }^{1}$ Patrick J. Smith, ${ }^{2}$ and Martin Grell ${ }^{1}$ \\ ${ }^{1}$ Department of Physics and Astronomy, University of Sheffield, Hicks Building, Hounsfield Rd, \\ Sheffield S3 7RH, United Kingdom \\ ${ }^{2}$ Laboratory of Applied Inkjet Printing, Department of Mechanical Engineering, Gladstone Building, \\ Garden Street, University of Sheffield, Sheffield S1 4BA, United Kingdom
}

(Received 15 March 2014; accepted 25 April 2014; published online 7 May 2014)

\begin{abstract}
We report the ionic liquid (IL) gating of a solution processed semiconducting polymer, poly(2,5bis(3-hexadecylthiophen-2-yl)thieno[3,2-b]thiophene) (PBTTT). IL gating relies on the poor solubility of PBTTT, which requires hot chlorinated benzenes for solution processing. PBTTT, thus, resists dissolution even in IL, which otherwise rapidly dissolves semiconducting polymers. The resulting organic thin film transistors (OTFTs) display low threshold, very high carrier mobility $\left(>3 \mathrm{~cm}^{2} / \mathrm{Vs}\right)$, and deliver high currents (in the order of $1 \mathrm{~mA}$ ) at low operational voltages. Such OTFTs are interesting both practically, for the addressing of current-driven devices (e.g., organic LEDs), and for the study of charge transport in semiconducting polymers at very high carrier density. (C) 2014 AIP Publishing LLC. [http://dx.doi.org/10.1063/1.4875746]
\end{abstract}

Much of the attraction of using organic semiconductors (OSCs) comes from their processing options: semiconducting polymers, in particular, can often be processed from solutions. To maximise processing advantages, all device components, not just the semiconductor, should be solution processed. A limiting factor, however, is the generally lower charge carrier mobility in OSCs, compared to conventional semiconductors. This is an issue in applications where organic thin film transistors (OTFTs) have to supply current, e.g., to drive organic light emitting diodes. Maximising the current delivered by solution-processed OTFTs is thus the subject of many studies, including this letter. Equation (1) provides a guideline towards high current OTFTs that work at moderate operational voltages

$$
I_{D, s a t}=\frac{W}{2 L} \mu C_{i}\left(V_{G}-V_{T}\right)^{2} .
$$

Wherein, the saturated drain current $I_{D, \text { sat }}$ is the highest current an OTFT can deliver at a given gate voltage, $\mathrm{V}_{\mathrm{G}} \cdot{ }^{1} \mathrm{~W} / \mathrm{L}$ stands for the width/length of the OTFT channel, $\mu$ for the semiconductor's charge carrier mobility, $C_{i}$ for the gate medium's specific capacitance, and $V_{T}$ for the OTFT threshold voltage. Clearly, high mobility semiconductors, combined with high capacitance gate media and low threshold maximise drain current for a given gate voltage. However, parameters $\mu, C_{i}$, and $V_{T}$ are convoluted, e.g., high $C_{i}$ leads to reduced $V_{T}$, but may rely on polar gate media that reduce $\mu .^{2}$ Also, every gate medium has a limited stability to applied voltages (dielectric breakdown for insulating gate media, electrochemical window for electrolytes). Hence, $V_{G}$ is limited to a practical maximum, $V_{G}(\max )$. As the geometry factor $W / L$ is somewhat arbitrary, we define two figures-ofmerit independent of $W / L$ to quantify the ability of an OTFT

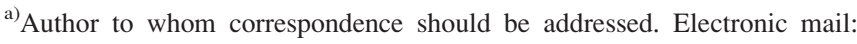
Php11saa@sheffield.ac.uk
}

to deliver current, namely the maximum sheet current $I_{W}$, and the lowest sheet resistance $R_{W}$, according to Eq. (2)

$$
\begin{gathered}
I_{W}=\frac{L}{W} I_{D, s a t}\left(V_{G}=V_{G}(\max )\right), \\
R_{W}=\frac{V_{G}(\max )}{I_{W}} .
\end{gathered}
$$

In recent years, OTFTs using electrolytes rather than insulators as gate media have been demonstrated, including solid electrolytes, ${ }^{3,4}$ water, ${ }^{5,6}$ and ionic liquids (ILs). ${ }^{7-9}$ Under applied gate voltage, an extremely thin electric double layer (EDL) forms at the electrolyte/semiconductor interface, with high specific capacitance $\left(>1000 \mathrm{nF} / \mathrm{cm}^{2}\right)$, low $V_{T}$, and consequently high $I_{W} /$ low $R_{W}$. Ionic liquids (ILs) lead to particularly high $C_{i}$, and display key practical advantages over aqueous electrolytes: ILs are not volatile, ${ }^{10}$ and IL/polymer formulations can be inkjet printed and subsequently gel into a quasi- solid film. ${ }^{11}$ However, ILs are excellent solvents, ${ }^{10}$ and thus not generally suited for use with solution processed OSCs- previous reports of IL- gated OTFTs used vacuum evaporated OSCs only. ${ }^{7-9}$

Here, we report on an OTFT that uses a solutionprocessed polymer OSC in conjunction with an IL gate medium. We used the polymer OSC poly(2,5-bis(3-hexadecylthiophen-2-yl)thieno[3,2-b]thiophene) (PBTTT), Fig. 1(a), which is known for its exceptionally high carrier mobility. ${ }^{4,12}$ Also, due to its particularly rigid conjugated backbone, PBTTT only dissolves in hot chlorinated benzenes. ${ }^{12} \mathrm{We}$ here show that PBTTT's poor solubility extends to an IL, 1ethyl-3-methylimidazolium-bis(trifluoromethyl-sulfonyl)imide ("EMITSFI") Fig. 1(b), which had previously been used as gate medium for evaporated OSCs. ${ }^{7,9}$ Resulting OTFTs display good figures- of- merit $I_{W}$ and $R_{W}$.

For OTFT fabrication, we used prefabricated gold source/drain (S/D) contact substrates of $\mathrm{W}=2 \mathrm{~mm} / \mathrm{L}=10 \mu \mathrm{m}$ $(W / L=200)$ described previously, ${ }^{6}$ and inkjet printed silver 


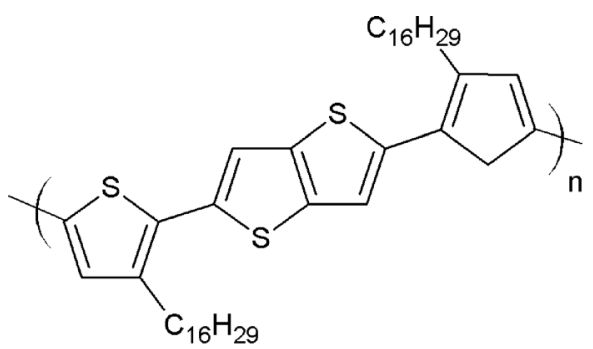<smiles>CCn1cc[n+](C)c1</smiles><smiles>O=S(=O)([N-]S(=O)(=O)C(F)(F)F)C(F)(F)F</smiles>

FIG. 1. Chemical structures of semiconducting polymer PBTTT, and ionic liquid EMITFSI.

(Ag) contacts of $W=3 \mathrm{~mm} / L=40 \mu \mathrm{m} \quad(W / L=75)$ printed from a $20 \mathrm{wt} \%$ suspension of silver nanoparticles (size ranged $20-50 \mathrm{~nm}$ ) in ethanol and ethylene glycol (from Sun Chemical, Slough, GB), similar to a procedure reported
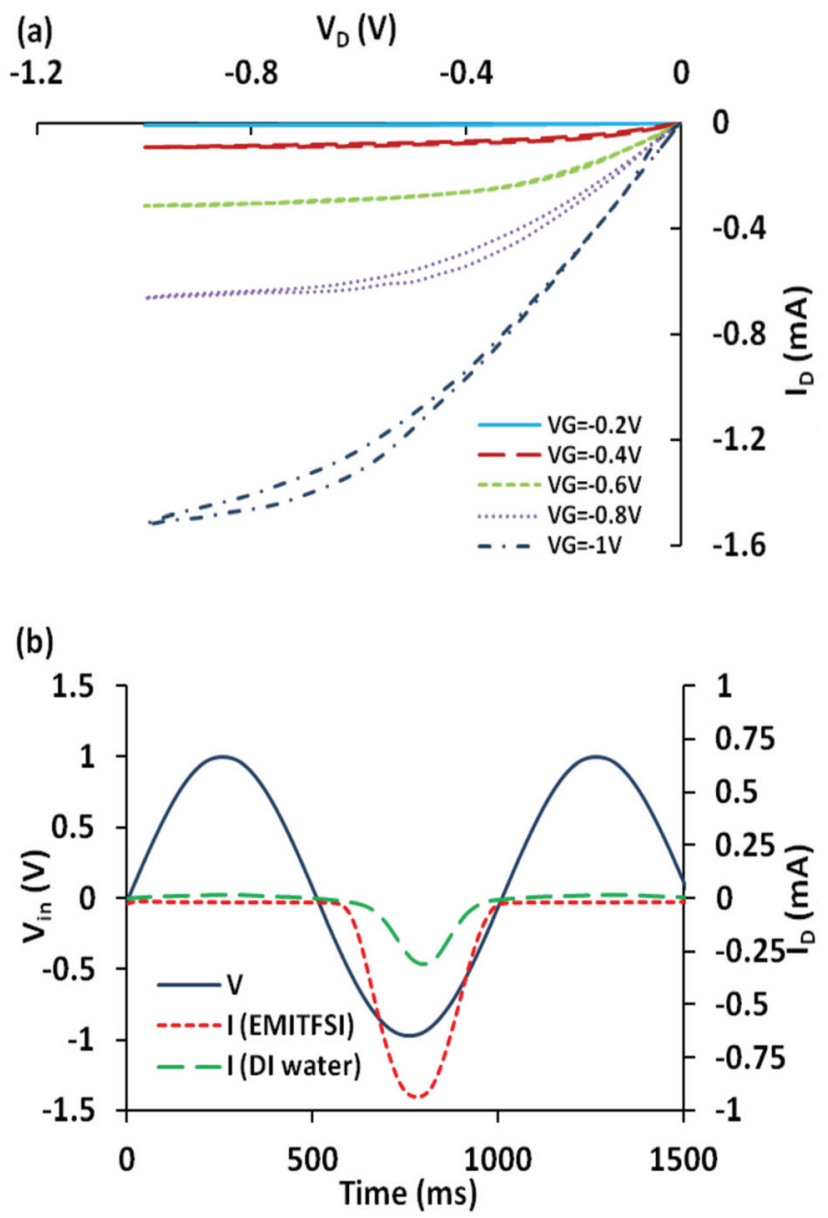

FIG. 2. (a) Output characteristics for IL- gated PBTTT OTFT with Au source/drain contacts. (b) Saturated transfer characteristics (red short dash), parameteric in time, for same device. Also shown is the saturated transfer characteristic for same device when gated with water (green long dash). The drive voltage, applied to the source, is shown in solid blue. ${ }^{16}$ previously. ${ }^{13,14}$ Ink viscosity and surface tension were $12 \mathrm{mPa}$ $\mathrm{s}$ and $27-31 \mathrm{mN} / \mathrm{m}$ at room temperature, respectively. We used a JetLab 4xl printing system (Microfab, Inc., Plano, TX), equipped with a $60 \mu \mathrm{m}$ diameter drop-on-demand piezoelectric printhead (MJ-AT-01-60, Microfab, Inc.). Printing frequency was set at $100 \mathrm{~Hz}$, voltage to $60 \mathrm{~V}$, and a pulse width to $10 \mu \mathrm{s}$. Printing was performed at room temperature onto glass substrates previously cleaned with acetone and isopropanol. Printed contact substrates were allowed to dry at room temperature for $10 \mathrm{~min}$ before being placed on a hotplate at $200^{\circ} \mathrm{C}$ for $60 \mathrm{~min}$ to remove the carrier solvent and organic components and sinter the silver nanoparticles. (An image of the printed S/D substrates is shown as an inset in Fig. 3(b)). The resistance between the pad for external contacting, and the "source" contact to the OTFT channel, was measured as $\sim 40 \Omega .7 \mathrm{mg} / \mathrm{ml}$ PBTTT sourced from Ossila Ltd was dissolved by stirring into hot 1,2 dichlorobenzene (DCB) $\left(110^{\circ} \mathrm{C}\right)$ for $45 \mathrm{~min}$, and spun onto contact substrates from hot $\left(100^{\circ} \mathrm{C}\right) \mathrm{DCB}$ for $40 \mathrm{~s}$ at $5000 \mathrm{rpm}$. After casting, films were dried under dynamic vacuum at $110^{\circ} \mathrm{C}$ for $1 \mathrm{~h}$. For electrolyte gating, we applied droplets $(5-8 \mu \mathrm{l})$ of EMITSFI IL (sourced from Aldrich), or DI water, from a microlitre syringe over the channel area, and inserted an $\mathrm{Au}$ gate needle (American Probe and Technologies) bent into L- shape. For
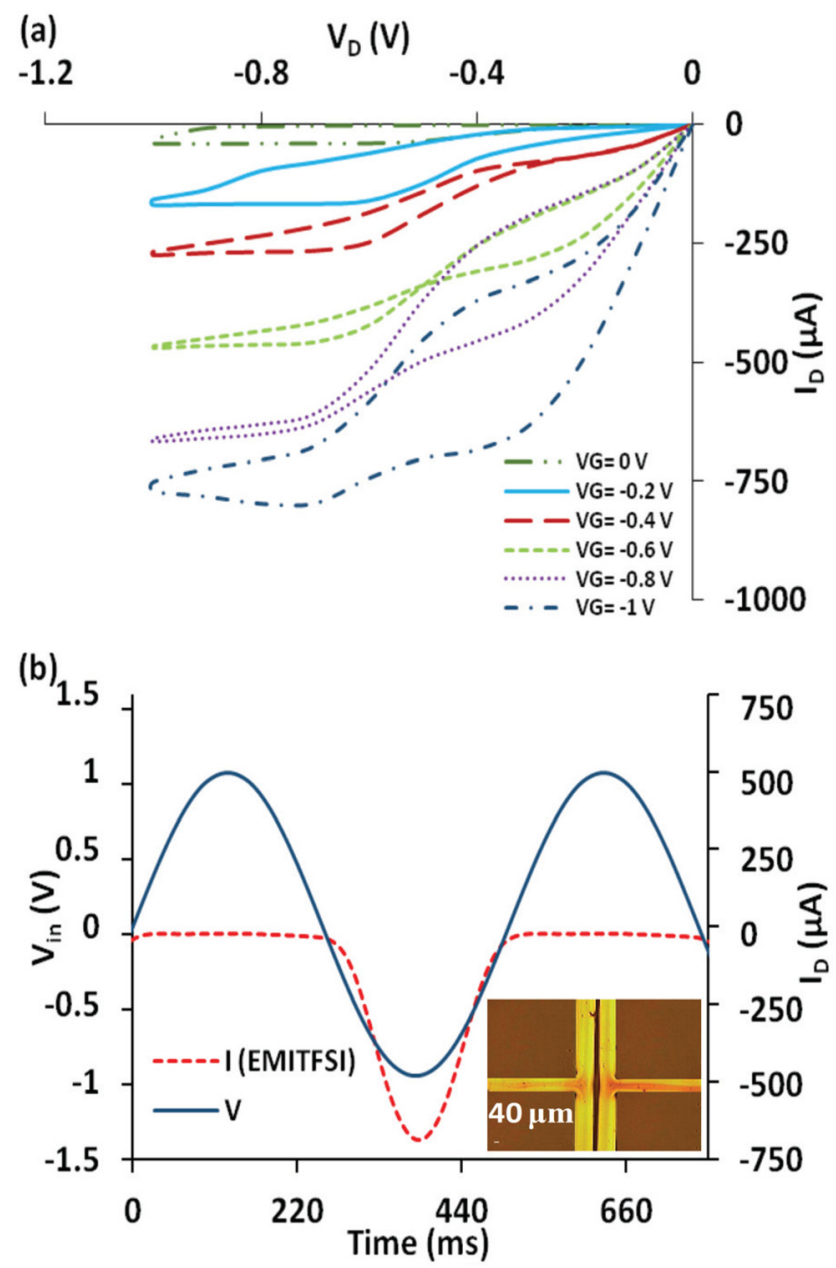

FIG. 3. (a) Output characteristics for IL- gated PBTTT OTFT with printed Ag source/drain contacts. (b) Saturated transfer characteristics (red short dash), parametric in time, for same device. Drive voltage in solid blue. Inset: Optical micrograph of printed/fused Ag contacts; $\mathrm{L}=40 \mu \mathrm{m}$. 
TABLE I. Figures- of- merit $I_{W}$ and $R_{W}$ (Eq. (2)) from recent publications, in comparison to data from this work. We include a range of semiconductors (solution processed and evaporated), and gate media (dielectrics, solid electrolytes, liquid electrolytes). We calculated $I_{W}$ and $R_{W}$ from characteristics shown in the respective publications.

\begin{tabular}{|c|c|c|c|c|c|c|}
\hline References & Semiconductor & Deposition & $\mathrm{h}^{+} / \mathrm{e}^{-}$ & Gate medium & $\boldsymbol{I}_{W}[\mu \mathrm{A} / \square]$ & $\boldsymbol{R}_{W}[\mathrm{M} \Omega / \square]$ \\
\hline Hasegawa et al. ${ }^{21}$ & $\mathrm{C}_{8}$-BTBT & Ink jet (2 shot) & $\mathrm{h}^{+}$ & Dielectric & 69 & 0.87 \\
\hline Klauk et $a .^{22}$ & DNTT & Evaporation & $\mathrm{h}^{+}$ & Dielectric & 0.54 & 5.56 \\
\hline Bao et al..$^{23}$ & Pentacene & Evaporation & $\mathrm{h}^{+}$ & Dielectric & 0.3 & 6.7 \\
\hline Horowitz et al..$^{5}$ & $\mathrm{P} 3 \mathrm{HT}$ & Spin casting & $\mathrm{h}^{+}$ & Water & 0.0054 & 111 \\
\hline Grell et al..$^{6}$ & ACN- conditioned BBL nanobelts & Drop casting & $\mathrm{e}^{-}$ & Water & 0.35 & 2.28 \\
\hline Berggren et al. $^{3}$ & $\mathrm{P} 3 \mathrm{HT}$ & Spin casting & $\mathrm{h}^{+}$ & Solid electrolyte & 0.0175 & 57 \\
\hline $\begin{array}{l}\text { Frisbie et al. }{ }^{3} \\
\text { Takeya et al. }{ }^{7}\end{array}$ & Rubrene & Evaporation & $\mathrm{h}^{+}$ & Ionic liquid & 0.66 & 0.3 \\
\hline Takeya et al..$^{9}$ & $\mathrm{C}_{60}$ & Evaporation & $\mathrm{e}^{-}$ & Ionic Liquid & 0.027 & 37 \\
\hline Heeney et $a l^{12}$ & PBTTT & Spin casting & $\mathrm{h}^{+}$ & Dielectric & 25 & 2.4 \\
\hline Torsi et al. ${ }^{4}$ & PBTTT & Spin casting & $\mathrm{h}^{+}$ & Solid electrolyte & 0.459 & 2.18 \\
\hline This work & PBTTT (Au contacts) & Spin casting & $\mathrm{h}^{+}$ & Ionic Liquid & 7.6 & 0.132 \\
\hline This work & PBTTT (inkjet Ag contacts) & Spin casting & $\mathrm{h}^{+}$ & Ionic Liquid & 9.7 & 0.102 \\
\hline This work & PBTTT (Au contacts) & Spin casting & $\mathrm{h}^{+}$ & Water & 1.6 & 0.64 \\
\hline
\end{tabular}

electrical characterisation, we used either a setup consisting of two Keithley 4200 source/measure units ${ }^{15}$ (for output characteristics), or a bespoke current/voltage (I/V) converter setup described previously ${ }^{6,16-18}$ for saturated transfer characteristics. All measurements were carried out under ambient atmosphere.

Figs. 2(a) and 2(b) shows output characteristics, and saturated transfer characteristics, of an IL- gated PBTTT OTFT with $\mathrm{Au}$ contacts. Note that the transfer characteristic is shown parametric in time, rather than explicitly. The acquisition and evaluation of parametric transfer characteristics, and the reasons for doing so, were discussed in detail previously. ${ }^{6,16-18}$ The PBTTT OTFT output characteristics display near- ideal shape, with little hysteresis and linear $I_{D}\left(V_{D}\right)$ behaviour for low $V_{D}$, which indicates good hole injection at the Au/PBTTT source contacts ("ohmic contacts"). At large negative $V_{D}$ and $V_{G}, I_{D}$ saturates; at $V_{G}=V_{D}=-1 V$, we observe a drain current of $\left|I_{D}\right|=1.52 \mathrm{~mA}$, which according to Eq. (2) gives $I_{W}=7.6 \mu \mathrm{A} / \square$ and $R_{W}=131.6 \mathrm{k} \Omega / \square$. This is an exceptionally low OTFT sheet resistance, in comparison to other recent reports, cited in Table I, of high performance OTFTs. Like Regioregular poly(3-hexylthiophene-2,5-diyl) (P3HT), ${ }^{5}$ PBTTT can also be gated with deionised water, an example is included in Fig. 2(b). Resulting drain currents are lower than for IL gating, but significantly larger than for water- gated P3HT. ${ }^{5}$

In a control experiment with IL on a blank contact substrate without OSC, we found maximum currents of only $0.45 \mu \mathrm{A}$. We can therefore safely exclude that parasitic currents in the IL account for the observed characteristics.

Charge carrier mobility in the saturated regime $\mu_{s a t}$, and threshold voltage $V_{T}$, can be evaluated from the saturated transfer characteristics in Fig. 2(b) by eliminating time, and plotting in the form $I_{D}^{1 / 2}$ vs $V_{G}{ }^{16}$ A straight line fit gives threshold from the intercept with the $V_{G^{-}}$axis, and $\mu_{\text {sat }}$ from the slope, $c f$. Eq. (1). We find $V_{T}=-0.36 \mathrm{~V}$ for IL gating. To evaluate $\mu_{\text {sat }}$, we assume specific capacitance of $C_{i} \sim 7000$ $\mathrm{nF} / \mathrm{cm}^{2}$, reported by Ono et al. ${ }^{7}$ for the IL EMITFSI at low frequency $(1 \mathrm{~Hz})$. We find $\mu_{\text {sat }}=3.24 \mathrm{~cm}^{2} / \mathrm{Vs}$, a remarkable carrier mobility for a polymer OSC. However, PBTTT is already known for its exceptional mobility, e.g., Hamadani et al. report a mobility of $\sim 1 \mathrm{~cm}^{2} / \mathrm{Vs}$ for dielectric- gated PBTTT OTFTs. ${ }^{12}$ In addition, gating with a high capacitance IL leads to very high charge carrier density in the accumulation layer, and it is known that mobility increases at high carrier density. ${ }^{19}$

When we measure saturated transfers on IL- gated PBTTT with drive voltages in excess of $1.2 \mathrm{~V}$, we soon find a decline in on/off ratio (on/off $\sim 2200$ under $1 \mathrm{~V}$ drive), and rapid decay in overall device performance. We believe this decline is related to the electrolysis of water: The highly hygroscopic IL will rapidly absorb water from atmospheric humidity, and while the "electrochemical window" of the EMITSFI IL used here is $4.3 \mathrm{~V},{ }^{10}$ it is only $1.23 \mathrm{~V}$ for water. We therefore limited ourselves to a maximum of $1 \mathrm{~V}$ in subsequent experiments. Limiting gate voltage to $1 \mathrm{~V}$ is not a serious drawback though: Xie and Frisbie have recently reported that mobility declines again in IL- gated devices at gate voltages $>1 \mathrm{~V},{ }^{8}$ probably due a binding between holes in the OSC and anions in the IL.

Realistic "printed electronics" requires solution processing of all OTFT components, including the S/D contacts. We have therefore also prepared IL-gated PBTTT OTFTs with printed Ag contacts. Characteristics are shown in Fig. 3.

Output characteristics Fig. 3(a) display significant hysteresis, and for low gate voltages, $I_{D}\left(V_{D}\right)$ at low drain voltage is not linear, but $\mathrm{I}_{D}$ remains sublinear initially and curves upwards only at higher $V_{D}$. Such sub- linear shape is the signature of a significant hole injection barrier at the printed $\mathrm{Ag} / \mathrm{PBTTT}$ contact, due to the lower work function of $\mathrm{Ag}$ compared to Au. The exceptionally high conductivity of the IL- gated channel exacerbates the poor contacts, leading to highly non-ideal output characteristics. However, at higher gate voltages, the injection problem at the contact is resolved, probably because the high gate voltage strongly assists tunnelling injection. Therefore, IL-gated PBTTT with printed Ag contacts deliver high drain currents at $V_{G}=V_{D}=-1 V$. In fact, sheet current is higher $\left(I_{W}=9.7 \mu \mathrm{A} / \square\right.$ vs. 
$\left.I_{W}=7.6 \mu \mathrm{A} / \square\right)$ and sheet resistance lower $\left(R_{W}=102 \mathrm{k} \Omega / \square\right.$ vs. $R_{W}=131.6 \mathrm{k} \Omega / \square$ ) than for OTFTs with Au S/D contacts, despite the injection barrier at the Ag contact. It is known, however, that apparent carrier mobility (and hence, sheet current), increases for longer channel length, L, because the relative contribution of contact resistance is diluted by longer channels, even when contacts are ohmic. ${ }^{20}$ The dependence of apparent mobility on L casts some doubt on the sheet current/sheet resistance metric in general, but it is nevertheless widely used for characterising conductive surfaces, e.g., synthetic metal films.

To summarise, we report the IL gating of a solution processed semiconducting polymer, PBTTT. Performance of resulting OTFT devices is shown in Table $\mathrm{I}$, in the form $I_{W}$ and $R_{W}$, in comparison to a few high- performance OTFTs from the recent literature.

Our figures-of-merit for entirely solution-processed OTFTs compare favourably within Table I, which is the result of the concurrent high capacitance, high carrier mobility, and low threshold, of the PBTTT/EMITSFI EDL transistor. Also, IL gating of PBTTT allows exploring the intriguing physics of OSCs at extremely high charge carrier concentrations $^{8}$ on the example of a semiconducting polymer. As a concluding remark, we note that currently, the operational lifetime of the devices introduced here is still limited to not much longer than $1 \mathrm{~h}$, probably due to an eventual slow dissolution of PBTTT in IL. Limited durability warrants further improvement, e.g., by the use of a gelled IL, ${ }^{11}$ or synthesis of a PBTTT derivative that can be crosslinked after deposition.

Saud Algarni and Talal M. Althagafi wish to thank the Cultural Attaché of Saudi Arabia to the UK and Taif University in Taif, Saudi Arabia for providing them with fellowships for their Ph.D. studies. The authors thank J. E. Macdonald and C. Dunscombe from Cardiff University, UK, for the provision of $\mathrm{Au}$ contact substrates.
${ }^{1}$ G. Horowitz, in Semiconducting Polymers, edited by G. Hadziioannou and P. F. Van Hutten (Wiley-VCH Verlag GmbH \& Co. KGaA, Weinheim, 2000), p. 463.

${ }^{2}$ J. Veres, S. D. Ogier, S. W. Leeming, D. C. Cupertino, and S. M. Khaffaf, Adv. Funct. Mater. 13(3), 199 (2003).

${ }^{3}$ E. Said, X. Crispin, L. Herlogsson, S. Elhag, N. D. Robinson, and M. Berggren, Appl. Phys. Lett. 89(14), 143507 (2006).

${ }^{4}$ L. M. Dumitru, K. Manoli, M. Magliulo, L. Sabbatini, G. Palazzo, and L. Torsi, ACS Appl. Mater. Interfaces 5(21), 10819 (2013).

${ }^{5}$ L. Kergoat, L. Herlogsson, D. Braga, B. Piro, M. Pham, X. Crispin, M. Berggren, and G. Horowitz, Adv. Mater. 22(23), 2565 (2010).

${ }^{6}$ A. Al Naim, A. Hobson, R. T. Grant, A. Dragoneas, M. Hampton, C. Dunscombe, T. Richardson, J. E. Macdonald, and M. Grell, Org. Electron. 14(4), 1057 (2013).

${ }^{7}$ S. Ono, S. Seki, R. Hirahara, Y. Tominari, and J. Takeya, Appl. Phys. Lett. 92(10), 103313 (2008).

${ }^{8}$ W. Xie and C. D. Frisbie, J. Phys. Chem. C 115(29), 14360 (2011).

${ }^{9}$ T. Uemura, M. Yamagishi, S. Ono, and J. Takeya, Appl. Phys. Lett. 95(10), 103301 (2009).

${ }^{10} \mathrm{R}$. Hagiwara and Y. Ito, J. Fluorine Chem. 105(2), 221 (2000).

${ }^{11}$ U. Löffelmann, N. Wang, D. Mager, P. J. Smith, and J. G. Korvink, J. Polym. Sci., Part B: Polym. Phys. 50(1), 38 (2012).

${ }^{12}$ B. H. Hamadani, D. J. Gundlach, I. McCulloch, and M. Heeney, Appl. Phys. Lett. 91(24), 243512 (2007).

${ }^{13}$ H. Meier, U. Löffelmann, D. Mager, P. J. Smith, and J. G. Korvink, Phys. Status Solidi A 206(7), 1626 (2009).

${ }^{14}$ D.-Y. Shin and P. J. Smith, J. Appl. Phys. 103(11), 114905 (2008).

${ }^{15}$ L. A. Majewski, R. Schroeder, M. Voigt, and M. Grell, J. Phys. D: Appl. Phys. 37(24), 3367 (2004).

${ }^{16}$ L. Hague, D. Puzzovio, A. Dragoneas, and M. Grell, Sci. Adv. Mater. 3, 907 (2011).

${ }^{17}$ L. Hague, D. Puzzovio, T. H. Richardson, and M. Grell, Sens. Lett. 9, 1692 (2011).

${ }^{18}$ A. F. Al Naim and M. Grell, J. Appl. Phys. 112(11), 114502 (2012).

${ }^{19}$ W. F. Pasveer, J. Cottaar, C. Tanase, R. Coehoorn, P. A. Bobbert, P. W. M. Blom, D. M. de Leeuw, and M. A. J. Michels, Phys. Rev. Lett. 94(20), 206601 (2005).

${ }^{20}$ E. C. P. Smits, S. G. J. Mathijssen, P. A. Van Hal, S. Setayesh, T. C. T. Geuns, K. A. H. A. Mutsaers, E. Cantatore, H. J. Wondergem, O. Werzer, and R. Resel, Nature 455(7215), 956 (2008).

${ }^{21}$ H. Minemawari, T. Yamada, H. Matsui, J. Tsutsumi, S. Haas, R. Chiba, R. Kumai, and T. Hasegawa, Nature 475(7356), 364 (2011).

${ }^{22}$ U. Zschieschang, F. Ante, T. Yamamoto, K. Takimiya, H. Kuwabara, M. Ikeda, T. Sekitani, T. Someya, K. Kern, and H. Klauk, Adv. Mater. 22(9), 982 (2010).

${ }^{23}$ M. E. Roberts, N. Queraltó, S. C. B. Mannsfeld, B. N. Reinecke, W. Knoll, and Z. Bao, Chem. Mater. 21(11), 2292 (2009). 